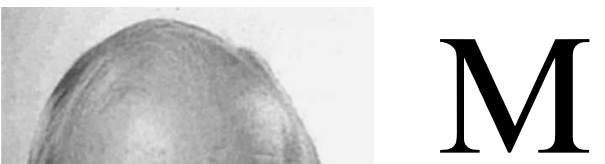

\title{
Slimming diets: Fakes and fraud
}

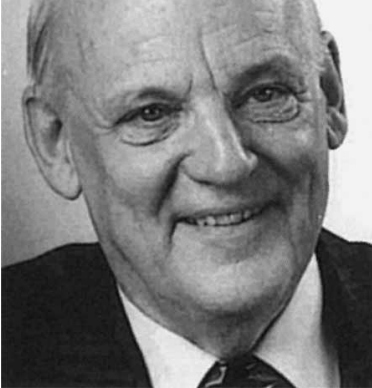

Gösta Samuelson gosta.samuelson@htu.se any overweight and obese people try to find different solutions to reduce their weight. With promises about rapid weight loss, many desperate and disillusioned people are lured into testing useless products, as emphasized by Dan Larhammar in an article in this issue of the Scandinavian Journal of Nutrition (SJN).

He comments critically upon different diets, e.g. the blood group diet. Many products advertised as excellent slimming diets are both expensive and not scientifically proven to be effective in a weight-reducing programme. Certain diets are also dangerous to health. The article is published together with others in SJN Issues 1 and 2, 2005, on the theme "Popular diets, body weight and health".

It is high time for medical experts and official authorities to take responsibility in evaluating and criticizing incorrect claims in the jungle of advertisements of slimming diets in mass media and elsewhere.

\section{Is coffee drinking beneficial or dangerous?}

Scandinavians are heavy coffee drinkers. It is, however, difficult to give a clear-cut answer as to whether caffeine has positive or negative effects on health in the long term. Some observational studies have shown a protective effect against type 2 diabetes, Parkinson's disease and Alzheimer's disease. Caffeine certainly has a stimulating effect on the central nervous system. However, as stated by Dag Thelle and Elisabeth Strandhagen in a review article in this issue, no relevant biological explanation for a protective effect against these diseases has been found. No firm conclusions can be drawn from studies published so far.

The effects of coffee on blood lipids and plasma homocysteine are more firmly based. Meta-analyses have shown a dose-response effect between coffee consumption and the levels of total cholesterol and low-density lipoprotein-cholesterol. Furthermore, heavy coffee drinking is positively associated with increased plasma homocysteine concentrations, which leads to an increased risk for cardiovascular disease.

In their own studies the authors found that four cups of filtered coffee daily led to an increase in plasma homocysteine, by $1 \mu \mathrm{mol} 1^{-1}$. Caffeine and chlorogenic acid probably account for the major part of the effect of coffee on total homocysteine levels. In addition, elevated homocysteine concentration is an independent factor for neural tube defects, pre-eclampsia, premature delivery, low birth weight, etc. In conclusion, the impact of excessive coffee drinking on long-term health remains unknown.

\section{Overweight and obesity: need for preventive approach}

Preventive measures are urgently needed to treat obesity. In 2002, the Swedish Council on Technology Assessment in Health Care (SBU) published the report "Obesity - a systematic literature review". Recently, an update of the report was published by SBU (No. 173, 2005, ISBN 91-85413-01-1). It is possible to prevent obesity if prevention starts in childhood, according to the report. Programmes are advocated for healthy diets and increased daily physical activity, starting in day-care centres and continuing at all school levels.

The WHO's Global Strategy on Diet, Physical Activity and Health, adopted in 2004, has begun intensive work globally, aiming to implement advice for better health. A basis for an Action Plan for Healthy Eating Habits and Increased Physical Activity, directed at several policy areas, has recently been presented to the Swedish Government by the Swedish National Food Administration and the National Swedish Institute of Health (www.slv.se).

In this context, the lifestyle of the family and the leisure time of adolescents should not to be forgotten. To reach young people, innovative methodological and pedagogic approaches are needed, preferably linked to scientific research projects. A powerful economic investment into longitudinal follow-up studies is vital. 